\title{
Análise Espacial de Fragmentos Florestais: Caso dos Parques Estaduais de Forno Grande e Pedra Azul, Estado do Espírito Santo
}

\author{
Ronie Silva Juvanhol ${ }^{1}$, Nilton Cesar Fiedler ${ }^{1}$, Alexandre Rosa dos Santos ${ }^{2}$ \\ Daiani Bernardo Pirovani ${ }^{1}$, Franciane Lousada Rubini de Oliveira Louzada ${ }^{1}$, \\ Henrique Machado Dias ${ }^{1}$, André Luiz Campos Tebaldi ${ }^{3}$ \\ ${ }^{1}$ Departamento de Engenharia Florestal, Universidade Federal do Espírito Santo - UFES \\ ${ }^{2}$ Departamento de Engenharia Rural, Universidade Federal do Espírito Santo - UFES \\ ${ }^{3}$ Instituto Estadual de Meio Ambiente e Recursos Hídricos - IEMA
}

\begin{abstract}
RESUMO
O objetivo foi analisar a estrutura da paisagem florestal entre duas Unidades de Conservação por meio de índices de ecologia da paisagem. O mapeamento foi obtido por digitalização de aerofoto, utilizando-se técnicas de fotointerpretação na escala 1:2500. No cálculo dos índices, foi utilizada Patch Analyst (ArcGis 9.3). Os fragmentos foram divididos em classes (muito pequeno: $<5$ ha; pequenos: $>5$ e $<10$ ha; médios: $>10$ e $<100$ ha; grandes: $>100$ ha). Foram calculados os índices de área, densidade, tamanho, forma, proximidade, área central (obtidos para diferentes simulações de efeito de borda: 40, 80, 100, $140 \mathrm{~m}$ ). Encontraram-se 2.652 fragmentos, representando $42,6 \%$ de cobertura florestal. Os fragmentos muito pequenos dominam (2.201), seguidos pelos médios (216), pequenos (177) e grandes (58). Houve diferenciações em relação às classes, demonstrando que os maiores apresentam métricas que indicam boa conservação. As classes média e grande indicam a necessidade de criação de políticas públicas que incentivem a formação de corredores ecológicos entre ambas as áreas protegidas.
\end{abstract}

Palavras-chave: conservação da natureza, ecologia da paisagem, geotecnologia.

\section{Spatial Analysis of Forest Fragments:}

\section{Case of the State Parks of Forno Grande and Pedra Azul, State of Espírito Santo}

\begin{abstract}
The objective of this work was to analyze the structure of forest landscape, between two conservation areas, through indices of landscape ecology. Mapping was obtained by scanning aerial photographs using photo-interpretation techniques at the scale of 1:2500. Patch Analyst (ArcGis 9.3) was used for the indices calculation. The fragments were divided in the following classes: very small $(<5 \mathrm{ha})$; small ( $>5$ and $<10 \mathrm{ha})$; medium ( $>10$ and $<100 \mathrm{ha})$; and large $(>100 \mathrm{ha})$. Indices of area, density, size, shape, proximity and central area were calculated; being obtained for different simulations of edge effect $(40,80,100,140 \mathrm{~m}) .2,652$ fragments were found, representing $42.6 \%$ of forest cover. The very small fragments were predominant $(2,201)$, followed by medium (216), small (177), and large fragments (58). There were differences in relation to classes, which demonstrated that the greatest present metrics showed good conservation. The middle and high classes indicated that public policies which encourage the creation of ecological corridors between the two protected areas should be generated.
\end{abstract}

Keywords: nature conservancy, landscape ecology, geotechnology. 


\section{INTRODUÇÃO}

O processo de fragmentação florestal é um fenômeno impulsionado pela atividade desordenada de uso e ocupação do território por meio de modelos econômicos exploratórios dos recursos naturais e pelos diferentes ciclos de crescimento e dispersão populacional (Tabarelli et al., 2010; Ribeiro et al., 2009; Tabarelli \& Gascon, 2005; Tabanez \& Viana, 2000; Nascimento et al., 1999). Esse fator tem feito com que a paisagem do bioma seja comprimida para pequenos fragmentos circundados por diferentes formas de matrizes do entorno (Tabarelli et al., 2010).

A Mata Atlântica brasileira não foge a essa regra, pois, desde a sua ocupação pelos colonizadores europeus, vem sofrendo com constantes processos de fragmentação florestal, oriundos dos diferentes usos do solo (Dean, 1996), pelos quais a alteração da paisagem primitiva, promovida pelo ser humano, é milhares de vezes maior do que a dinâmica de perturbação natural do ecossistema (Tabarelli \& Gascon, 2005).

Ao mesmo tempo em que a Mata Atlântica é considerada uma das florestas mais ameaçadas do planeta (Myers et al., 2000), também é considerada como um dos biomas mais importantes e prioritários para a conservação da biodiversidade, pois abriga muitas espécies endêmicas e vulneráveis à extinção (Silva et al., 2007), além de complexos vegetacionais que têm na sua ecologia funcional papel primordial para a manutenção dos ecossistemas presentes nesse bioma (Scarano, 2009).

Esse bioma se apresenta como um mosaico de vegetação, que originalmente cobria 150 milhões de ha e, por consequência do processo de fragmentação, está reduzido aos atuais 11,7\% (Ribeiro et al., 2009). O Estado do Espírito Santo está todo coberto pelo bioma Mata Atlântica (Pereira, 2007); no entanto, atualmente conta com aproximadamente $11 \%$ do original (Fundação... e Instituto..., 2009). Diante disso, é necessária maior atenção no que diz respeito às políticas de conservação, principalmente as referentes à gestão do entorno das áreas protegidas para a formação de corredores ecológicos; estes constituem uma forma de amortecer os fragmentos relevantes, pois a perda de ambientes naturais aliada ao processo de fragmentação tem resultado na formação de paisagens com pouca riqueza biológica (Fahrig, 2003).

As barreiras ocasionadas pela fragmentação diminuem a dispersão dos organismos entre os remanescentes florestais, resultando em mudanças na estrutura e na função das comunidades florestais (Tabarelli et al., 2010). Sendo assim, é fundamental o desenvolvimento de ações voltadas para promover a conexão entre fragmentos (Metzger et al., 2009). Louzada (2010) afirma que o corredor entre os Parques Estaduais de Forno Grande e Pedra Azul encontra-se em área de domínio da Mata Atlântica, o qual apresenta pressão antrópica em seu entorno a partir de atividades desordenadas e sem planejamento de uso e ocupação do território. Nesse sentido, ações que revertam e/ou minimizem essa situação tornam-se necessárias para promover a sustentabilidade ambiental no uso do bioma.

Para avaliar o grau de efetividade evulnerabilidade dos corredores ecológicos, é necessário avaliar os padrões estruturais, espaciais e temporais dos fragmentos florestais. Nesse sentido, o uso de métricas e índices de ecologia da paisagem justificase para promover um zoneamento no emprego desses recursos para a manutenção da biodiversidade, pois a análise dessas métricas permite auxiliar na definição de técnicas de manejo visando a recuperar e/ou conservar os remanescentes florestais.

Nesse sentido, o objetivo desse estudo foi mapear e analisar a estrutura da paisagem florestal no corredor ecológico entre os Parques Estaduais de Forno Grande e Pedra Azul, região serrana do Estado do Espírito Santo e, por meio de índices métricos da paisagem, selecionar áreas aptas para a conservação ambiental e o uso sustentável dos recursos naturais associados ao planejamento territorial promovido pela ecologia da paisagem.

\section{MATERIAL E MÉTODOS}

\subsection{Caracterização da área de estudo}

A área de estudo está localizada na região serrana no sul do Estado do Espírito Santo (Figura 1). O Parque Estadual de Forno Grande possui 770 ha e localiza-se no município de Castelo $\left(20^{\circ} 30^{\prime} 35^{\prime \prime}\right.$; 


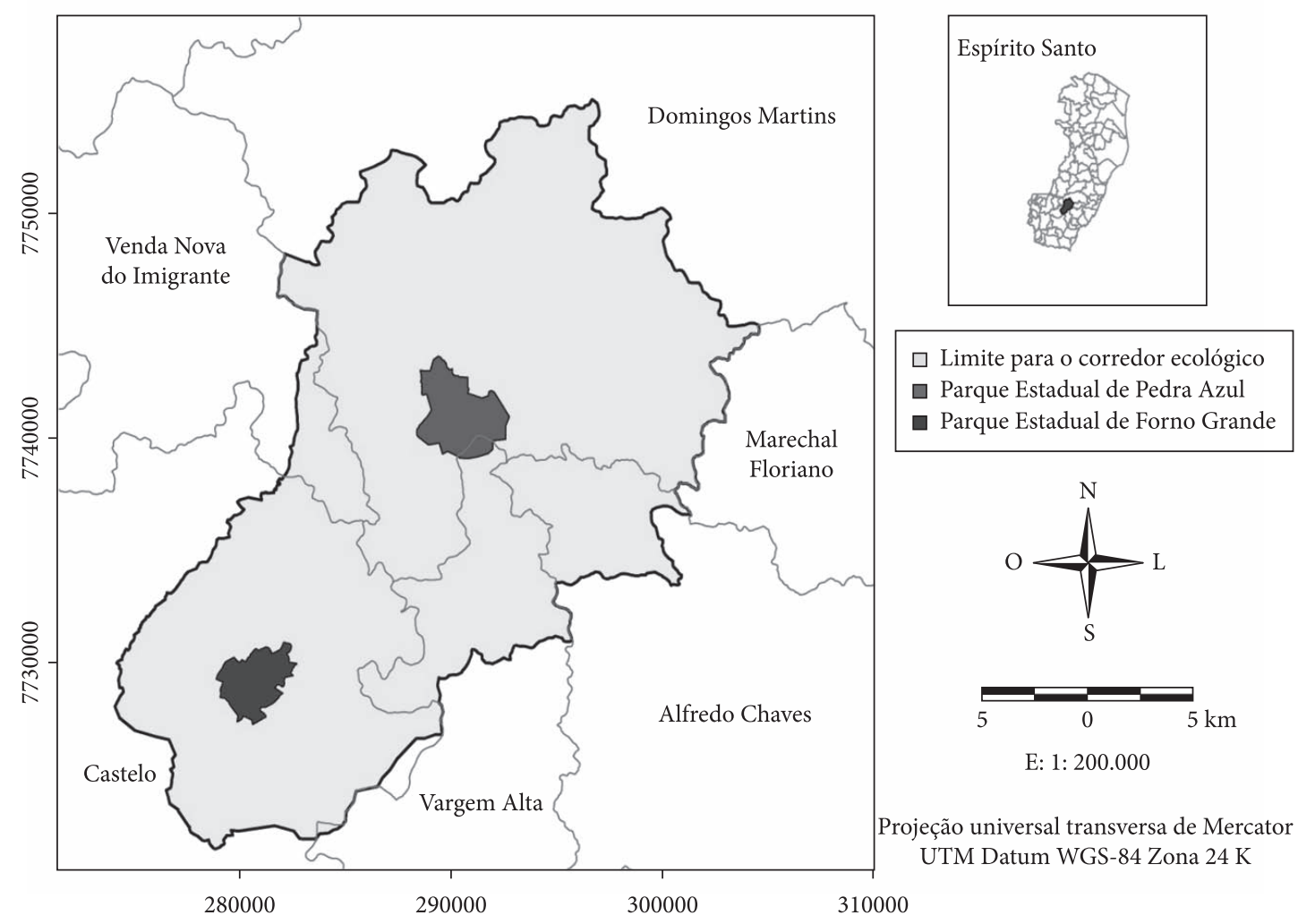

Figura 1. Limite do corredor ecológico entre os Parques Estaduais de Forno Grande e Pedra Azul, no Estado do Espírito Santo.

Figure 1. Limit to the ecological corridor between State Parks of Forno Grande and Pedra Azul, in Espírito Santo State.

$20^{\circ} 32^{\prime} 29^{\prime \prime}$ S e $41^{\circ} 05^{\prime} 20^{\prime \prime} ; 41^{\circ} 07^{\prime} 17^{\prime \prime}$ O). O Parque Estadual de Pedra Azul possui 1.196 ha e localizase no município de Domingos Martins (20²3' 36"; $20^{\circ} 25^{\prime} 56^{\prime \prime} \mathrm{S}$ e $40^{\circ} 55^{\prime} 23^{\prime \prime} ; 41^{\circ} 01^{\prime} 40^{\prime \prime} \mathrm{O}$ ). O corredor ecológico entre ambas as unidades está concentrado em uma área de $511,21 \mathrm{~km}^{2}$, que abrange os municípios de Domingos Martins, Venda Nova do Imigrante, Castelo, Vargem Alta e Alfredo Chaves. Os Parques Estaduais de Forno Grande e Pedra Azul ocupam 1,5 e 2,3\% do corredor ecológico, respectivamente. Essa região está determinada pelo Ministério do Meio Ambiente (Brasil, 2002) como área prioritária para conservação, além de também estar inserida na Reserva da Biosfera da Mata Atlântica do Estado do Espírito Santo. A vegetação da região pertence ao domínio da Floresta Ombrófila Densa Montana e Altomontana, com transição para a Floresta Estacional Semidecidual (Veloso et al., 1991). Associados ao ambiente florestal, também estão localizados ecossistemas rupestres em razão dos afloramentos rochosos abundantes na região (Louzada, 2010).

\subsection{Mapeamento dos fragmentos florestais}

As etapas metodológicas utilizadas na elaboração do mapa de fragmentação florestal estão representadas no fluxograma da Figura 2. O mapa dos fragmentos florestais da área de estudo foi obtido por meio da digitalização da aerofoto da região, em escala de 1:35000, disponibilizada pelo Instituto Estadual de Meio Ambiente e Recursos Hídricos (IEMA). A digitalização via tela foi feita na escala padrão de 1:2500 no aplicativo computacional ArcGis 9.3, por meio das técnicas de fotointerpretação. Em caso de dúvidas, procedeu-se à ampliação do campo visual da imagem até uma escala de 1:5000 para verificação e posterior digitalização, sendo elaborado um arquivo vetorial poligonal para a classe de fragmento florestal (Louzada, 2010). 


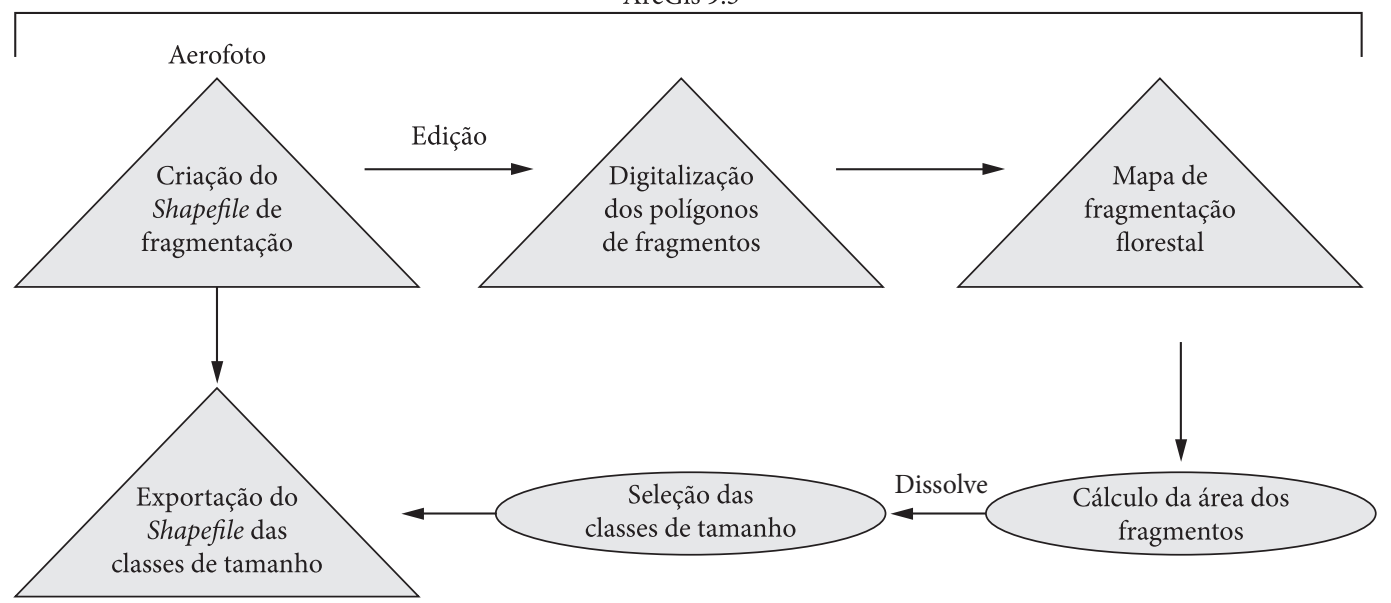

Figura 2. Etapas metodológicas para elaboração do mapa de fragmentação florestal.

Figure 2. Stages of the methodology used to elaboration the map of forest fragmentation.

A partir do mapa de fragmentação florestal, foi quantificada a área de cada fragmento usando-se a calculadora de valores da tabela de atributos do próprio arquivo de imagem vetorial poligonal, o que tornou possível comparar os tamanhos dos diversos fragmentos florestais encontrados no corredor ecológico. O número de fragmentos existentes na área foi relacionado à classe de tamanho às quais pertencem: muito pequenos ( $<5 \mathrm{ha})$, pequenos (5-10 ha), médios (10-100 ha) e grandes (>100 ha).

\subsection{Mapeamento dos fragmentos florestais por meio de métricas da paisagem}

A análise dos fragmentos foi feita com base no mapeamento de fragmentos florestais préestabelecido neste estudo, com o intuito de se obter o número de fragmentos, a relação de tamanho e formato entre eles, e o grau de proximidade, por meio dos índices de ecologia da paisagem. Os índices ou métricas da paisagem para os fragmentos foram obtidos dentro do aplicativo computacional ArcGis 9.3, por meio da extensão gratuita Patch Analyst (Analisador de Manchas).

Os arquivos de fragmentação florestal com as diferentes classes de tamanho, no formato shapefile ou vetorial, foram transformados para o formato raster ou matricial e, posteriormente, foram calculadas as métricas, utilizando-se a versão para dados matriciais (Patch Grid). No sentido de se evitar a utilização de métodos redundantes, optou-se por utilizar as seguintes métricas: índices de densidade de fragmentos; tamanho; forma dos fragmentos; índices de borda; área central, e índice de proximidade.

Para o cálculo do índice de área central, utilizaram-se as distâncias de 40, 80, 100 e 140 m de borda, obtendo-se assim diferentes cenários para as análises. As métricas de forma exigem a adoção de uma paisagem padrão para efeito de comparação. Nesse sentido, a forma dos fragmentos foi comparada ao formato de um quadrado para o cálculo da razão perímetro/borda, sendo os valores de índice de forma mais próximos de um, ligados a formatos mais regulares. $\mathrm{O}$ detalhamento das métricas utilizadas neste estudo, juntamente com suas siglas e significados, pode ser visualizado na Tabela 1.

\section{RESULTADOS E DISCUSSÃO}

\subsection{Mapeamento dos fragmentos florestais}

O mapeamento das áreas de florestas possibilitou contabilizar 2.652 fragmentos florestais em toda a área representativa do limite do corredor ecológico. Esse número corresponde a uma área de $21.749,80$ ha de remanescentes florestais, significando que, da área do corredor (51.100 ha), 42,6\% são fragmentos florestais. Esse predomínio de cobertura florestal na área de estudo demonstra o estado de preservação 
Tabela 1. Índices de ecologia da paisagem gerados no nível de classes por meio do Patch Analyst junto aos fragmentos florestais.

Table 1. Indices of landscape ecology generated at the level of classes by the Patch Analyst to forest fragments.

\begin{tabular}{|c|c|c|c|c|}
\hline Grupo & Sigla & Métrica & Unidade & Observação \\
\hline Área & CA & Área da Classe & Hectare (ha) & $\begin{array}{l}\text { Somatório das áreas de todas as manchas ou } \\
\text { de fragmentos florestais presentes na área. }\end{array}$ \\
\hline \multirow{4}{*}{$\begin{array}{l}\text { Densidade e } \\
\text { tamanho }\end{array}$} & MPS & $\begin{array}{l}\text { Tamanho médio da } \\
\text { mancha }\end{array}$ & Hectare (ha) & $\begin{array}{l}\text { Soma do tamanho das manchas dividido pelo } \\
\text { número de manchas. }\end{array}$ \\
\hline & NUMP & Número de manchas & Adimensional & Número total de manchas na paisagem/classe. \\
\hline & PSSD & $\begin{array}{l}\text { Desvio padrão do ta- } \\
\text { manho da mancha }\end{array}$ & Hectare (ha) & Razão da variância do tamanho das manchas. \\
\hline & PSCoV & $\begin{array}{l}\text { Coeficiente de variação } \\
\text { do tamanho da mancha }\end{array}$ & $\begin{array}{l}\text { Porcentagem } \\
(\%)\end{array}$ & $\begin{array}{l}\text { Desvio padrão do tamanho da mancha } \\
\text { dividido pelo tamanho médio da mancha, } \\
\text { multiplicado por } 100 \text {. }\end{array}$ \\
\hline \multirow{2}{*}{ Borda } & $\mathrm{TE}$ & Total de bordas & Metro (m) & $\begin{array}{l}\text { Extremidade total de todas as manchas, sendo } \\
\text { a soma de perímetro de todas as manchas }\end{array}$ \\
\hline & $\mathrm{ED}$ & Densidade de borda & $\mathrm{m} / \mathrm{ha}$ & $\begin{array}{l}\text { Quantidade de extremidades relativa à área da } \\
\text { paisagem. }\end{array}$ \\
\hline \multirow{3}{*}{ Forma } & MSI & Índice de forma médio & Adimensional & $\begin{array}{l}\text { É igual a um quando todas as manchas } \\
\text { forem circulares e aumenta com a crescente } \\
\text { irregularidade da forma da mancha. }\end{array}$ \\
\hline & AWMSI & $\begin{array}{l}\text { Índice de forma de área } \\
\text { média ponderada }\end{array}$ & Adimensional & $\begin{array}{l}\text { Difere do MSI, porque manchas maiores terão } \\
\text { mais peso que as menores. }\end{array}$ \\
\hline & MPFD & $\begin{array}{l}\text { Dimensão fractal da } \\
\text { mancha média }\end{array}$ & Adimensional & $\begin{array}{l}\text { Os valores se aproximam de um para formas } \\
\text { com perímetros simples e chega a dois quando } \\
\text { as formas forem mais complexas. }\end{array}$ \\
\hline \multirow{6}{*}{ Área central } & TCA & Área Central Total & Hectare (ha) & O tamanho total das manchas centrais. \\
\hline & MCA & Área central média & Hectare (ha) & $\begin{array}{l}\text { O tamanho médio das manchas de área } \\
\text { central. }\end{array}$ \\
\hline & NCA & Número de áreas centrais & Adimensional & $\begin{array}{c}\text { Número total de áreas centrais dentro da } \\
\text { paisagem ou dentro de cada mancha no nível } \\
\text { de classe. }\end{array}$ \\
\hline & CASD & $\begin{array}{l}\text { Desvio padrão de área } \\
\text { central }\end{array}$ & Hectare (ha) & Raiz da variância da média das áreas centrais. \\
\hline & $\mathrm{CACoV}$ & $\begin{array}{l}\text { Coeficiente de variação } \\
\text { de área central }\end{array}$ & $\begin{array}{l}\text { Porcentagem } \\
\quad(\%)\end{array}$ & $\begin{array}{c}\text { Representa a variabilidade em tamanho das } \\
\text { áreas centrais disjuntas em relação à área } \\
\text { central média. }\end{array}$ \\
\hline & TCAI & $\begin{array}{c}\text { Índice de Área Central } \\
\text { Total }\end{array}$ & $\begin{array}{l}\text { Porcentagem } \\
(\%)\end{array}$ & $\begin{array}{l}\text { Medida da quantidade relativa de área central } \\
\text { na paisagem. }\end{array}$ \\
\hline Proximidade & $\mathrm{MNN}$ & $\begin{array}{l}\text { Distância média do } \\
\text { vizinho mais próximo }\end{array}$ & $\operatorname{Metros}(\mathrm{m})$ & $\begin{array}{l}\text { A distância média do vizinho mais próximo } \\
\text { é a média dessas distâncias para classes } \\
\text { individuais no nível de classe e a distância } \\
\text { média da classe vizinha mais próxima no nível } \\
\text { de paisagem. }\end{array}$ \\
\hline
\end{tabular}

Fonte: Adaptado de MacGarigal \& Marks (1995) e Pirovani (2010).

da região, ressaltando a importância da realização de estudos e ações que beneficiem a preservação e a conservação das espécies da região.

O mapeamento dos fragmentos florestais demonstrou que a maior parte dos remanescentes da Mata Atlântica é caracterizada por fragmentos muito pequenos (0-5 ha), correspondendo a $83 \%$ do número total de fragmentos florestais encontrados
(2.201). Saunders et al. (1991) afirmam que a riqueza diminui quando a área do fragmento fica menor do que as áreas mínimas necessárias para a sobrevivência das populações.

A classe dos fragmentos pequenos (5-10 ha) representou um percentual de 6,7\% do número total de fragmentos, com 177 fragmentos, e a classe dos médios (10-100 ha) representou 8,1\% do 
total de fragmentos florestais mapeados, com 216 fragmentos.

A classe dos fragmentos grandes, composta por aqueles acima de 100 ha, representou um baixo valor $(2,2 \%)$ em relação ao número total de fragmentos florestais, com 58 fragmentos. No entanto, cabe ressaltar que esses fragmentos maiores correspondem a $58,8 \%$ da área total. Esse elevado percentual é caracterizado pelo fragmento florestal que interliga os Parques Estaduais de Forno Grande e Pedra Azul, possuindo 8.592,94 ha. A Figura 3 ilustra a distribuição das diferentes classes de tamanho dos fragmentos florestais na área de estudo.

Os fragmentos muito pequenos e pequenos, em relação à área total dos fragmentos florestais, são de apenas $14,7 \%$, juntamente com o alto número de fragmentos presentes nas referidas classes de tamanho, com 2.378 fragmentos. Segundo Lima (1997), a ocorrência de grande quantidade de pequenos fragmentos florestais é comum em paisagens de Mata Atlântica. O principal problema é a área estar susceptível a alterações na dinâmica da paisagem, como as ações do efeito de borda (Tabanez \&S Viana, 2000).

$\mathrm{Na}$ análise dos fragmentos florestais por meio de métricas da paisagem, os índices de ecologia da paisagem para as classes de tamanho e para todos os fragmentos da área encontram-se na Tabela 2.

A relação do número de fragmentos e da área que estes ocupam na maioria das vezes é inversa, ou seja, os grandes fragmentos possuem menor percentual em número, porém representam uma parcela maior da área total dos remanescentes florestais mapeados. Os fragmentos pequenos possuem maior número de unidades. No entanto, a soma de suas áreas representa menor percentual da área total dos fragmentos florestais mapeados.

A média de tamanho para todos os fragmentos analisados conjuntamente, sem distinção de diferentes classes de tamanho, é de 10,54 ha. O desvio padrão do tamanho da mancha (PSSD) é de 200,4 ha. Isso indica a existência de fragmentos com valores de área muito acima e/ou muito abaixo do valor médio, como pode ser verificado para a classe

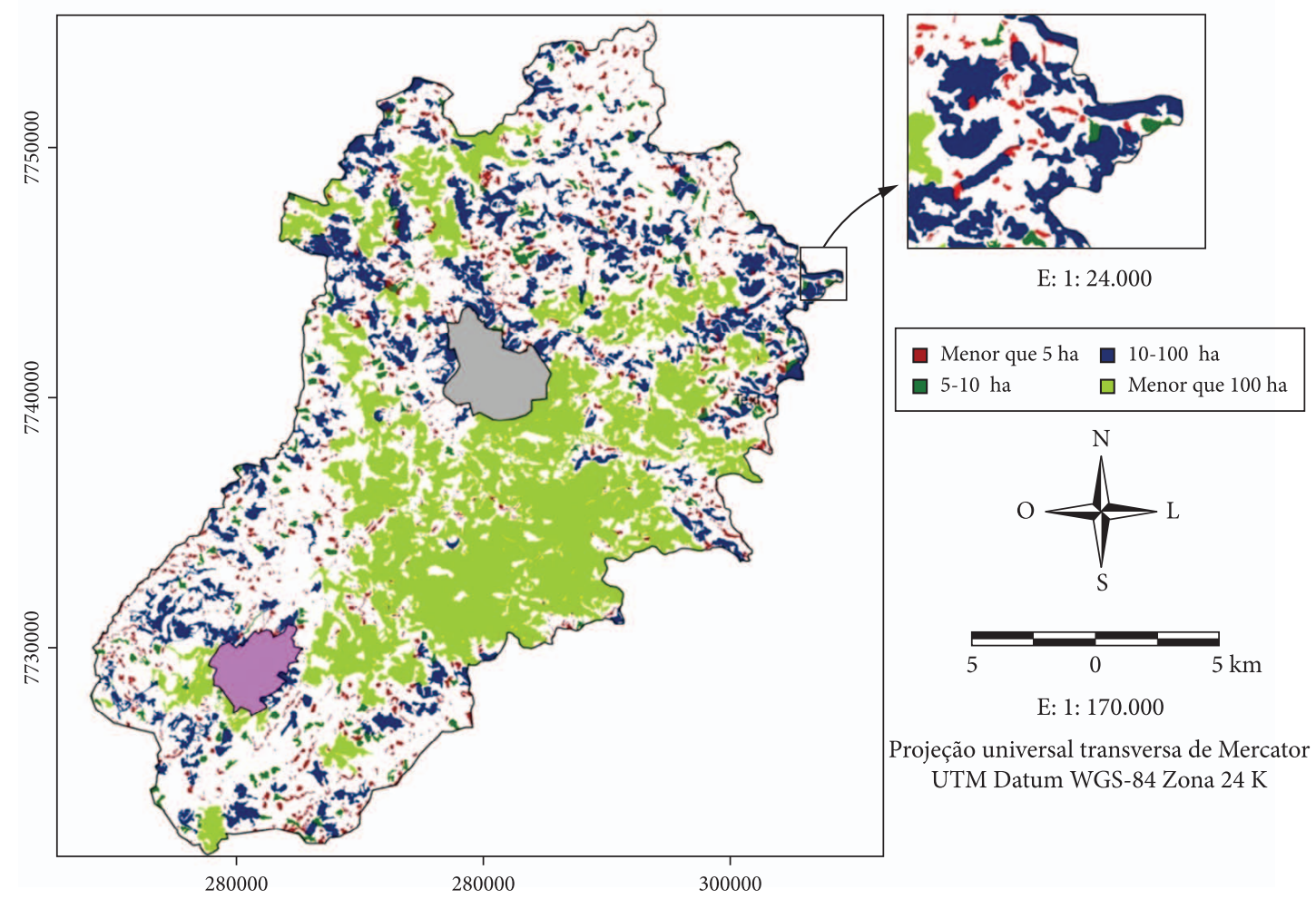

Figura 3. Distribuição das classes de tamanho dos fragmentos florestais na área de estudo.

Figure 3. Distribution of size classes of forest fragments in the study area. 
Tabela 2. Índices de ecologia da paisagem calculados para os fragmentos florestais no limite do corredor ecológico entre os Parques Estaduais de Forno Grande e Pedra Azul, ES.

Table 2. Indices of landscape ecology calculated for the forest fragments at the limit of the ecological corridor between the State Parks of Forno Grande and Pedra Azul, ES.

\begin{tabular}{|c|c|c|c|c|c|c|c|}
\hline \multirow[b]{2}{*}{ Grupo } & \multirow[b]{2}{*}{ Índices } & \multirow[b]{2}{*}{ Unidade } & \multicolumn{5}{|c|}{ Classes de tamanho } \\
\hline & & & $\begin{array}{l}\text { Muito pequeno } \\
(<5 \text { ha })\end{array}$ & $\begin{array}{l}\text { Pequeno } \\
(5-10 \text { ha) }\end{array}$ & $\begin{array}{c}\text { Médio } \\
(10-100 \text { ha })\end{array}$ & $\begin{array}{c}\text { Grande } \\
(>100 \text { ha })\end{array}$ & $\begin{array}{c}\text { Todos } \\
(0,1 \mathrm{a}>100 \mathrm{ha})\end{array}$ \\
\hline Área & $\mathrm{CA}$ & Hectares (ha) & $1.937,9$ & $1.249,0$ & $5.776,2$ & $12.786,7$ & $21.749,80$ \\
\hline \multirow{4}{*}{$\begin{array}{l}\text { Densidade e } \\
\text { tamanho }\end{array}$} & MPS & Hectares (ha) & 0,88 & 7,06 & 26,74 & 220,4 & 10,54 \\
\hline & NUMP & Adimensional & 2.201 & 177 & 216 & 58 & 2652 \\
\hline & PSSD & Hectares (ha) & 1,34 & 3,71 & 28,56 & 111,62 & 200,4 \\
\hline & PSCoV & Porcentagem & 152,31 & 52,51 & 106,80 & 506,34 & 1900,51 \\
\hline \multirow{2}{*}{ Borda } & $\mathrm{TE}$ & $\operatorname{Metros}(\mathrm{m})$ & $1.157 .026,0$ & $395.060,0$ & $1.150 .032,0$ & $1.229 .670,0$ & $3.743 .922,0$ \\
\hline & ED & $\mathrm{m} / \mathrm{ha}$ & 597,0 & 287,4 & 199,1 & 96,1 & 172,1 \\
\hline \multirow{3}{*}{ Forma } & MSI & Adimensional & 1,56 & 1,93 & 2,52 & 2,8 & 1,71 \\
\hline & AWMSI & Adimensional & 1,92 & 2,03 & 3,26 & 13,68 & 11,35 \\
\hline & MPFD & Adimensional & 1,10 & 1,11 & 1,14 & 1,12 & 1,10 \\
\hline Proximidade & MNN & Metros (m) & 894,3 & 446,9 & 174,8 & 167,3 & 413,3 \\
\hline
\end{tabular}

CA (Área de todas as manchas da classe); MPS (Tamanho médio da mancha); NumP (Número de manchas); PScoV (Coeficiente de variação do tamanho da mancha); PSSD (Desvio padrão do tamanho da mancha); MSI (Índice de forma médio); AWMSI (Índice de forma de área média ponderada); MPFD (Dimensão fractal da mancha média); TE (Total de bordas); ED (Densidade de borda); MNN (Distância média do vizinho mais próximo).

de tamanho grande, com média de tamanho de fragmentos de 220,4 ha e PSSD de 111,6 ha.

As métricas de borda revelaram menor valor (TE) para a classe dos fragmentos pequenos $(395.060,0 \mathrm{~m})$, ao contrário dos fragmentos grandes, que apresentaram o maior valor total $(1.229 .670,0 \mathrm{~m})$. Já a classe de fragmentos muito pequenos foi constituída de menor quantitativo total de borda $(1.157 .026,0 \mathrm{~m})$ do que os fragmentos grandes. Porém, ao comparar esse valor de borda com sua contribuição em área, que é bem menor do que os fragmentos grandes, percebeu-se maior proporção borda/área nos fragmentos menores. Para os fragmentos médios (1.150.032,0 m), observaramse valores similares aos muito pequenos.

Os fragmentos muito pequenos e pequenos apresentaram maior densidade de bordas (ED), com soma de $883,8 \mathrm{~m}$ de borda por hectare contra $199,1 \mathrm{~m} /$ ha dos fragmentos médios e $96,2 \mathrm{~m} / \mathrm{ha}$ dos fragmentos grandes. Essa diferença da quantidade de bordas, quando se considera a densidade, devese aos valores de área ocupados por cada classe de tamanho dos fragmentos, sendo a densidade de bordas inversamente proporcional à área ocupada por cada classe. Esses resultados apontam para um menor efeito de borda nos fragmentos grandes, indicando maior grau de conservação.
Em fragmentos menores, onde a transição entre o fragmento florestal e a matriz é muito abrupta, cria-se uma borda que expõe a floresta às condições encontradas nas adjacências. A porção externa da floresta adjacente à borda torna-se parte da zona de transição, ocasionando mudanças microclimáticas, como o aumento da temperatura e o estresse hídrico, acarretando alterações na composição de espécies e na estrutura da vegetação. Nesse sentido, o grau de interferência que tal fragmentação causa no ecossistema, como o aumento do efeito de borda, é consequência desse processo e leva a uma constância na redução das florestas, ocasionando maior vulnerabilidade para redução da riqueza e abundância de espécies adultas e plântulas (Ribeiro et al., 2009; Portela \& Santos, 2007; Tabanez \& Viana, 2000).

Além do tamanho, a forma dos fragmentos também influencia no grau do impacto do efeito de borda (Farina, 1998). A comparação dos valores de índice de forma (MSI) das classes de tamanho dos fragmentos florestais revelou que os fragmentos muito pequenos e pequenos apresentaram formato mais regular (MSI $=1,53$ e 1,93, respectivamente) quando comparados com os fragmentos médios e grandes, que apresentaram valores de MSI de 2,52 e 2,8, respectivamente. Assim, os formatos 
mais irregulares aumentaram com o tamanho dos fragmentos. No entanto, apesar de os índices de forma indicarem formatos mais irregulares para os fragmentos grandes e mais regulares para os fragmentos pequenos, o tamanho e a forma do fragmento estão intrinsecamente ligados à borda, pois quanto menor o fragmento ou mais alongado, mais intenso será o efeito de borda, diminuindo a razão interior-margem (Primack \& Rodrigues, 2001). Assim, mesmo apresentando formatos mais irregulares, os fragmentos maiores estão sob menor efeito de borda do que os menores.

$\mathrm{O}$ índice de forma médio ponderado pela área (AWMSI) para as classes de tamanho dos fragmentos florestais são superiores ao observado para o índice de forma médio (MSI), indicando que os fragmentos de maior área têm formas mais irregulares que a média. Essa relação existe porque, para o cálculo do índice de forma médio ponderado pela área (AWMSI), os fragmentos receberam pesos em função de seu tamanho.

O grau de isolamento dos fragmentos, expresso pela distância média do vizinho mais próximo (MNN), apresentou resultado semelhante para as classes de tamanho médio e grande, estando apenas os fragmentos de tamanho médio com isolamento de $174,8 \mathrm{~m}$ e os fragmentos grandes com distância de isolamento de $167,3 \mathrm{~m}$.

Nesse contexto, pode-se considerar que os fragmentos para as classes de tamanho médio e grande possuem baixo grau de isolamento, ao se comparar com os fragmentos muito pequenos e pequenos, que apresentaram uma distância de isolamento maior (896 e 446,9 m, respectivamente). Embora em maior número, a classe de fragmentos muito pequenos apresentou maior isolamento entre vizinhos mais próximos.

Ao analisar a distância entre todos os tamanhos de fragmentos ( $\mathrm{MNN}=413,3 \mathrm{~m}$ ), o grau de isolamento decresce. Nesse sentido, é importante considerar os fragmentos de menor tamanho como trampolins ecológicos nessa paisagem. $\mathrm{O}$ isolamento depende das distâncias e áreas dos fragmentos vizinhos, além do arranjo espacial desses fragmentos.

As métricas relativas às áreas centrais (núcleo) dos fragmentos estão expressas na Tabela 3, objetivando investigar qual faixa de borda exerce maior influência na área central dos fragmentos. Para uma borda de $40 \mathrm{~m}$, os fragmentos muito pequenos apresentam área central total de 235,5 ha, que correspondem a um índice de área central total (TCAI) de $12,16 \%$. Isso significa que, da área total dos fragmentos muito pequenos (1937,9 ha), apenas esse total corresponde à área de interior. Logo, 87,8\% da área total dos fragmentos muito pequenos está sob o efeito de borda de $40 \mathrm{~m}$. Para esse valor de efeito de borda, é encontrado o número de áreas centrais (NCA) entre os fragmentos muito pequenos de 623. Dividindo-se a área central total por esse número, tem-se uma área central média (MCA)

Tabela 3. Índices de ecologia da paisagem relativos às métricas de área central.

Table 3. Indices of landscape ecology relative the metrics to central area.

\begin{tabular}{|c|c|c|c|c|c|c|c|c|c|c|c|c|}
\hline \multirow{2}{*}{$\begin{array}{c}\text { Distância } \\
\text { de borda } \\
(\mathrm{m})\end{array}$} & \multicolumn{6}{|c|}{ Muito pequeno ( $<5$ ha) } & \multicolumn{6}{|c|}{ Pequeno (5-10 ha) } \\
\hline & TCA & TCAI & CACoV & CASD & NCA & MCA & TCA & TCAI & CACoV & CASD & NCA & MCA \\
\hline 40 & 235,5 & 12,16 & 142,03 & 0,54 & 623 & 0,38 & 456,5 & 36,55 & 108,52 & 2,03 & 244 & 1,87 \\
\hline 80 & 91,3 & 0,47 & 112,9 & 0,16 & 65 & 1,4 & 957,9 & 7,67 & 152,09 & 0,81 & 180 & 5,3 \\
\hline 100 & 0,08 & 0 & 56,57 & 0,02 & 3 & 0,03 & 207,9 & 1,66 & 198,03 & 0,54 & 76 & 2,7 \\
\hline 140 & 0 & 0 & 0 & 0 & 0 & 0 & 20,3 & 0,16 & 158,49 & 0,64 & 5 & 4,1 \\
\hline \multirow{2}{*}{$\begin{array}{l}\text { Distância } \\
\text { de borda } \\
(\mathrm{m})\end{array}$} & \multicolumn{6}{|c|}{ Médio (10-100 ha) } & \multicolumn{6}{|c|}{ Grande (>100 ha) } \\
\hline & TCA & TCAI & CACoV & CASD & NCA & MCA & TCA & TCAI & CACoV & CASD & NCA & MCA \\
\hline 40 & 3050 & 52,8 & 229,29 & 13,4 & 523 & 5,83 & 9701,6 & 75,9 & 1064,1 & 465,04 & 222 & 43,7 \\
\hline 80 & 1412,9 & 24,46 & 262,09 & 8 & 463 & 3,05 & 7332,4 & 57,3 & 1214,3 & 349,17 & 255 & 28,75 \\
\hline 100 & 789,9 & 13,67 & 263,95 & 6,19 & 337 & 2,34 & 6086,6 & 47,6 & 1285,5 & 297,51 & 263 & 23,14 \\
\hline 140 & 310,9 & 5,38 & 252,53 & 4,54 & 173 & 1,8 & 4663,7 & 36,5 & 1248 & 262,18 & 222 & 21,01 \\
\hline
\end{tabular}

TCA (Área central total); TCAI (Índice de área central total); CACoV (Coeficiente de variação de área central); CASD (Desvio padrão de área central); NCA (Número de áreas centrais); MCA (Área central média). 
com valor muito baixo para fins de conservação, que chega a 0,38 ha. Ao analisar o efeito de borda de $40 \mathrm{~m}$ para a classe de fragmento de tamanho pequeno, observam-se padrões semelhantes em relação à classe de fragmentos de tamanho muito pequeno. Entretanto, o efeito de borda tende a diminuir em função do aumento da área central média.

A área central total (TCA) dos fragmentos médios, considerando o efeito de borda de $40 \mathrm{~m}$, é de 3050 ha, apresentando valor maior para o referido índice quando comparado aos fragmentos muito pequenos e pequenos. A porcentagem de área dessa classe ocupada pelas áreas interiores, expressa pelo TCAI, é de 52,8\%, representando um menor efeito de borda (47,2\%), quando comparada aos fragmentos menores. Observa-se que o valor de número de áreas centrais (NCA) é de 523, maior que o total de fragmentos médios (216), o que se deve ao fato de alguns fragmentos, em função da irregularidade de seu formato, apresentarem mais de uma área central. O mesmo acontece com os fragmentos pequenos e grandes, sendo o NCA encontrado para a borda de $40 \mathrm{~m}$ correspondente a 244 e 222 áreas centrais, respectivamente, enquanto que para a classe de tamanho pequeno, com apenas 177 fragmentos, e para a classe grande, 58 fragmentos. Assim, considerando o menor efeito de borda para os fragmentos grandes, o total de área central (TCA) é de 9.701,6 ha e o TCAI corresponde a 75,9\%, sendo o valor de área central média (MCA), para essa situação, de 43,7 ha.

A Figura 4 demonstra que a variação do TCAI (em porcentagem) aumenta para a distância de borda de $80 \mathrm{~m}$. Os fragmentos muito pequenos e pequenos tiveram redução mais considerável das métricas de áreas centrais, diminuindo o valor de TCAI para $0,47 \%$ e $7,67 \%$, respectivamente. Os fragmentos médios reduziram o índice de área central pela metade, passando a $24,46 \%$, enquanto que os fragmentos grandes sofreram menor redução com a borda (57,3\%).

A distância de borda de $100 \mathrm{~m}$ levou as métricas de área central dos fragmentos muito pequenos a resultados nulos, sendo o TCAI de $0,0 \%$ e o valor de TCA de 0,08 ha, representados por uma redução do valor de NCA de apenas três áreas centrais. Em uma distância de borda de $140 \mathrm{~m}$, tais fragmentos deixarão

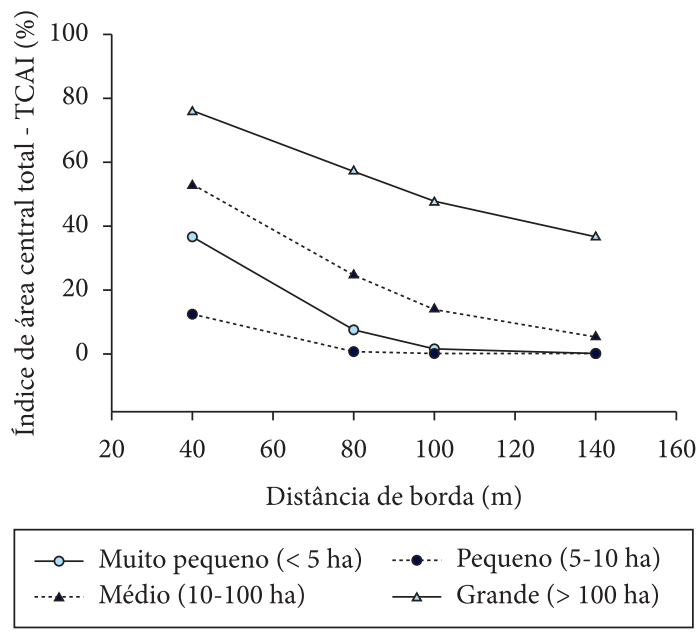

Figura 4. Comportamento do índice de área central total com o aumento da distância de borda para as classes de tamanho dos fragmentos florestais.

Figure 4. Behavior of the central area index with the increase distance from the edge to size classes of forest fragments.

de existir. Isso significa que, sob tais condições, essa classe de tamanho encontra-se totalmente dominada pelo efeito de borda, estando todos os fragmentos susceptíveis à influência completa da matriz.

O TCAI dos fragmentos médios para as distâncias de borda de 100 e $140 \mathrm{~m}$ foi de 13,67 e $5,38 \%$, respectivamente. Nos fragmentos maiores, o aumento na distância de borda leva a diminuições nas métricas de áreas centrais menores e mais gradativas. $\mathrm{O}$ índice de área central total diminuiu de $47,6 \%$, quando a borda considerada foi de $100 \mathrm{~m}$, para $36,5 \%$, quando se considerou a faixa de borda de $140 \mathrm{~m}$. Nota-se que, para essa classe de tamanho, a simulação com a maior distância de borda não leva à dominação do efeito de borda sobre os fragmentos, diferentemente dos fragmentos muito pequenos $\mathrm{e}$ pequenos.

As influências do efeito de borda podem ser diversas, dependendo da espécie e de sua resposta às variações ocorridas no ambiente - como incidência luminosa, maior exposição ao vento, presença de espécies exóticas (invasoras ou não), umidade e temperatura heterogênea -, em que ambas afetarão a produtividade primária do ecossistema por meio das diferenças microclimáticas e das interações ecológicas (Portela \& Santos, 2007; Alves Junior et al., 2006; Paciencia \& Prado, 2004). Nesse 


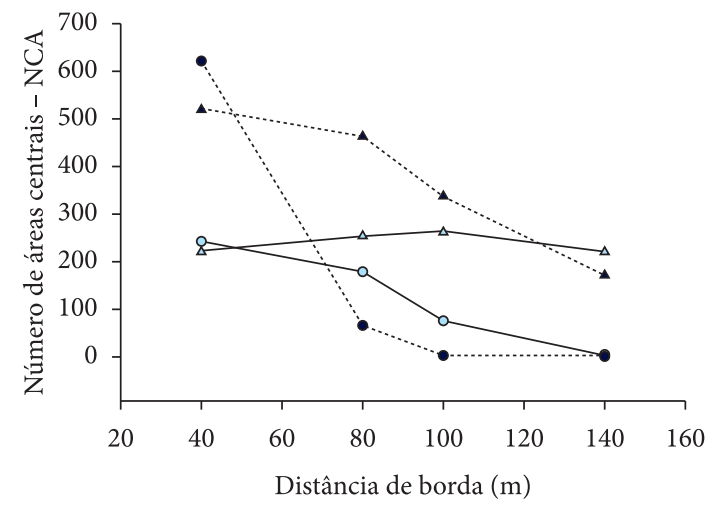

$\multimap$ Muito pequeno (<5 ha) $\quad \ldots . \cdots$ Pequeno (5-10 ha)

…-... Médio (10-100 ha) $\quad \longrightarrow$ Grande (>100 ha)

Figura 5. Comportamento do número de áreas centrais com o aumento da distância de borda para as classes de tamanho dos fragmentos florestais.

Figure 5. Behavior of the number of areas central with the increase distance from the edge to size classes of forest fragments.

sentido, para detectar causa e efeito do processo de fragmentação florestal, associado à dinâmica de borda, é necessário inferir sobre a estrutura comunitária do mesmo, a partir da mensuração da riqueza e da abundância dessa faixa, evidenciando impactos, como a diminuição do fragmento, a partir da taxa de mortalidade e da abundância de espécies.

O número de áreas centrais decresce com o aumento da distância de borda, exceto entre as distâncias de 80 a $100 \mathrm{~m}$ na classe de fragmentos grandes. No entanto, para os fragmentos muito pequenos tal redução é mais abrupta para a distância de 40 a $80 \mathrm{~m}$, enquanto que, nos fragmentos pequenos e médios, o NCA decai, proporcionalmente, com o aumento das faixas de borda (Figura 5).

A Figura 6 demonstra que os fragmentos grandes apresentaram valores de área central média (MCA) muito superiores às demais classes de tamanho. Já as demais classes de tamanho, apresentaram valores próximos. Isso pode estar associado à explicação para os valores apresentados de MCA no grupo dos fragmentos grandes por estarem na presença de fragmentos muito superiores, também expressa pelo alto desvio padrão da mancha $(\mathrm{CASD}=111,62 \%)$, anteriormente demonstrado na Tabela 2.

Os resultados das métricas de área central apontam para o fato de que quanto maior o tamanho

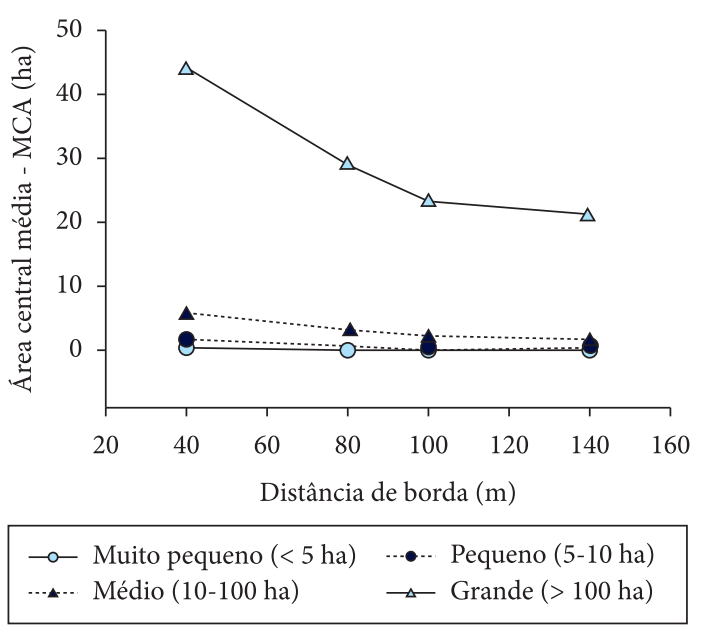

Figura 6. Comportamento da área central média com o aumento da distância de borda para as classes de tamanho dos fragmentos florestais.

Figure 6. Average behavior of the central area with the increase distance from the edge to size classes of forest fragments.

do fragmento, maior será sua área central total (TCA) e menor sua área afetada pelo efeito de borda. Com a fragmentação excessiva, ocorre aumento da área da borda em relação à área do fragmento. Por isso, a relação perímetro/área aumenta e, se o fragmento continua a diminuir de tamanho, pode acabar se tornando inteiramente um habitat de borda, como acontece com os fragmentos menores detectados nesse corredor ecológico entre os Parques de Forno Grande e Pedra Azul.

\section{CONCLUSÕES}

De acordo com os resultados apresentados, pode-se concluir que os fragmentos florestais nessa área representativa do corredor ecológico são representados, em sua maioria, por fragmentos menores que 5 ha, indicando um alto grau de fragmentação florestal. Além disso, quanto mais irregulares são os formatos, maior será o total de bordas dos fragmentos, e quanto maior o tamanho do fragmento, menor será a influência do efeito de borda. Os índices de proximidade para as classes de tamanho médio e grande indicam positivamente a criação de políticas públicas que incentivem a formação de corredores ecológicos entre essas Unidades de Conservação. 


\section{STATUS DA SUBMISSÃO}

Recebido: 02/08/2011

Aceito: 03/10/2011

Resumo publicado online: 04/10/2011

Artigo completo publicado: 22/12/2011

\section{AUTOR(ES) PARA CORRESPONDÊNCIA}

\section{Nilton Cesar Fiedler}

Departamento de Engenharia Florestal, Universidade Federal do Espírito Santos - UFES

Av. Governador Lindemberg, 316, Centro, CEP 29550-000

Jerônimo Monteiro, ES, Brasil

e-mail: fiedler@pq.cnpq.br

\section{Henrique Machado Dias}

Departamento de Engenharia Florestal, Universidade Federal do Espírito Santos - UFES, Av. Governador Lindemberg, 316, Centro, CEP 29550-000

Jerônimo Monteiro, ES, Brasil

e-mail: henridias@yahoo.com.br

\section{REFERENNCIAS}

Alves Junior FT, Brandão CFLS, Rocha KD, Marangon LC, Ferreira RLC. Efeito de borda na estrutura de espécies arbóreas em um fragmento de Floresta Ombrófila Densa, Recife, PE. Revista Brasileira de Ciências Agrárias 2006; 1: 49-56.

Brasil. Ministério do Meio Ambiente - MMA. Biodiversidade brasileira: avaliação e identificação de áreas e ações prioritárias para conservação, utilização sustentada e repartição de benefícios da biodiversidade brasileira. Brasília: MMA; 2002.

Dean W. A ferro e fogo: a história e a devastação da Mata Atlântica brasileira. São Paulo: Companhia das Letras; 1996.

Fahrig L. Effects of habitat fragmentation on biodiversity. Annual Review of Ecology, Evolution and Systematic 2003; 34: 487-515. http://dx.doi.org/10.1146/ annurev.ecolsys.34.011802.132419

Farina A. Principles and methods in landscape ecology. Londres: Chapman \& Hall; 1998.

Fundação SOS Mata Atlântica, Instituto Nacional de Pesquisas Espaciais - INPE. Atlas dos remanescentes florestais da Mata Atlântica entre o período 2005-2008. São Paulo: INPE; 2009. Relatório Parcial.
Lima EACF. Estudo da paisagem do município de Ilha Solteira-SP: subsídios para planejamento físicoambiental [tese]. São Carlos: Universidade Federal de São Carlos; 1997.

Louzada FLRO. Proposta de Corredores Ecológicos para interligação dos Parques Estaduais de Forno Grande e Pedra Azul, ES, utilizando geotecnologias [dissertação]. Alegre: Universidade Federal do Espírito Santo; 2010.

Macgarigal K, Marks BJ. Fragstats: Spatial pattern analysis program for quantifying landscape structure. Corvallis: Oregon State University; 1995. Reference manual.

Metzger JP, Martensen AC, Dixo M, Bernacci LC, Ribeiro MC, Teixeira AMG et al. Time-lag in biological responses to landscape changes in a highly dynamic Atlantic Forest region. Biological Conservation 2009; 142: 1166-1177. http://dx.doi. org/10.1016/j.biocon.2009.01.033

Myers N, Mittermeier RA, Mittermeier CG, Fonseca GAB, Kent J. Biodiversity hotspots for conservation priorities. Nature 2000; 403: 853-845. PMid:10706275. http://dx.doi.org/10.1038/35002501

Nascimento HEM, Dias AS, Tabanez AAJ, Viana VM. Estrutura e dinâmica de populações arbóreas de um fragmento de floresta estacional semidecidual na região de Piracicaba, SP. Revista Brasileira de Biologia 1999; 59(2): 329-342. http://dx.doi.org/10.1590/ S0034-71081999000200015

Paciencia MLB, Prado J. Efeitos de borda sobre a comunidade de pteridófitas na Mata Atlântica da região de Una, sul da Bahia, Brasil. Revista Brasileira de Botânica 2004; 27(4): 641-653.

Pereira OJ. A cobertura vegetal do Espírito Santo. In: Simonelli M, Fraga CN, organizadores. Espécies da flora ameaçada de extinção do estado do Espírito Santo. Vitória: IPEMA; 2007.

Pirovani DB. Fragmentação florestal e dinâmica da ecologia da paisagem na bacia hidrográfica do Rio Itapemirim [dissertação] Alegre: Universidade Federal do Espírito Santo; 2010.

Portela RCQ, Santos FAM. Produção e espessura da serapilheira na borda e interior de fragmentos florestais de Mata Atlântica de diferentes tamanhos. Revista Brasileira de Botânica 2007; 30(2): 271-280.

Primack RB, Rodrigues E. Biologia da Conservação. Londrina: E. Rodrigues; 2001.

Ribeiro MC, Metzger JP, Martensen AC, Ponzoni FJ, Hirota MM. The Brazilian Atlantic Forest: How much is left, and how is the remaining forest distributed? Implications for conservation. Biological conservation 2009; 142: 1141-1153. http://dx.doi. org/10.1016/j.biocon.2009.02.021 
Saunders DA, Hobbs RJ, Margules CR. Biological consequences of ecosystem fragmentation: a review. Conservation Biology 1991; 5(1): 18-35. http://dx.doi. org/10.1111/j.1523-1739.1991.tb00384.x

Scarano FR. Plant communities at the periphery of the Atlantic rain forest: rare-species bias and its risks for conservation. Biological Conservation 2009; 142: 12011208. http://dx.doi.org/10.1016/j.biocon.2009.02.027

Silva WGS, Metzger JP, Simões S, Simonetti C. Relief influence on the spatial distribution of the Atlantic Forest cover at the Ibiúna Plateau, SP. Brazilian Journal of Biology 2007; 67: 403-411. http://dx.doi.org/10.1590/ S1519-69842007000300004

Tabanez AAJ, Viana VM. Patch structure within Brazilian Atlantic Forest fragments and implications for conservation. Biotropica 2000; 32(4b): 925-933. http://
dx.doi.org/10.1646/0006-3606(2000)032[0925:PSWBA $\mathrm{F}] 2.0 . \mathrm{CO} ; 2$

Tabarelli M, Aguiar AV, Ribeiro MC, Metzger JP, Peres CA. Prospects for biodiversity conservation in the Atlantic Forest: lessons from aging human-modified landscapes. Biological Conservation 2010; 143: 23282340. http://dx.doi.org/10.1016/j.biocon.2010.02.005

Tabarelli M, Gascon C. Lessons from fragmentation research: improving management and policy guidelines for biodiversity conservation. Conservation Biology 2005; 19(3): 734-739. http://dx.doi.org/10.1111/ j.1523-1739.2005.00698.x

Veloso HP, Rangel-Filho ALR, Lima JCA. Classificação da vegetação brasileira, adaptada a um sistema universal. Rio de Janeiro: IBGE; 1991. 